\title{
Results with radiative events from KLOE
}

\author{
Debora Leone* on behalf of the KLOE collaboration ${ }^{\dagger}$ \\ Institut für Experimentelle Kernphysik - University of Karlsruhe, Germany \\ E-mail: debora.leone@iekp.fzk.de
}

The KLOE experiment at the electron-positron collider DA $\Phi N E$ has recently proven the feasibility of using initial state radiation (ISR) in $e^{+} e^{-}$annihilation for precision measurements of hadronic cross section. This new method, dubbed Radiative Return, allows us to measure hadronic cross sections for energies $M^{2}$ (hadron) $<s$ at particle factories which operate at fixed centre-of-mass energy $s$. In the recently published KLOE measurement of the pion form factor, events had been collected where the ISR photon is emitted at small polar angles, which reduces the background from final state radiation (FSR) but leads to a suppression of the threshold region $M_{\pi \pi}^{2}<0.35 \mathrm{GeV}^{2}$. We present here a complementary analysis using events with ISR photons emitted at large polar angles. Photon tagging is possible in this case (contrary to the small photon polar angle analysis) and the threshold region becomes accessible. Preliminary results are presented, as well as a measurement of the forward-backward asymmetry, which allows a test of the FSR model used for the description of the photon radiation from pions.

International Europhysics Conference on High Energy Physics

July 21 st - 27th 2005

Lisboa, Portugal

\footnotetext{
*Speaker.

${ }^{\dagger}$ F. Ambrosino, A. Antonelli, M. Antonelli, C. Bacci, P. Beltrame, G. Bencivenni, S. Bertolucci, C. Bini, C. Bloise, V. Bocci, F. Bossi, D. Bowring, P. Branchini, R. Caloi, P. Campana, G. Capon, T. Capussela, F. Ceradini, S. Chi, G. Chiefari, P. Ciambrone, S. Conetti, E. De Lucia, P. De Simone, G. De Zorzi, S. Dell'Agnello, A. Denig, A. Di Domenico, C. Di Donato, S. Di Falco, B. Di Micco, A. Doria, M. Dreucci, G. Felici, A. Ferrari, M. L. Ferrer, G. Finocchiaro, C. Forti, P. Franzini, C. Gatti, P. Gauzzi, S. Giovannella, E. Gorini, E. Graziani, M. Incagli, W. Kluge, V. Kulikov, F. Lacava, G. Lanfranchi, J. Lee-Franzini, D. Leone, M. Martini, P. Massarotti, W. Mei, S. Meola, S. Miscetti, M. Moulson, S. Müller, F. Murtas, M. Napolitano, F. Nguyen, M. Palutan, E. Pasqualucci, A. Passeri, V. Patera, F. Perfetto, L. Pontecorvo, M. Primavera, P. Santangelo, E. Santovetti, G. Saracino, B. Sciascia, A. Sciubba, F. Scuri, I. Sfiligoi, T. Spadaro, M. Testa, L. Tortora, P. Valente, B. Valeriani, G. Venanzoni, S. Veneziano, A. Ventura, R. Versaci, G. Xu
} 


\section{Introduction}

The hadronic contribution to the magnetic anomaly of the muon $a_{\mu}=\left(g_{\mu}-2\right) / 2$ is related to the measurement of the cross section $e^{+} e^{-} \rightarrow$ hadrons via the dispersion integral

$$
a_{\mu}(\text { hadr })=\frac{1}{4 \pi^{3}} \int_{4 m_{\pi}^{2}}^{\infty} \sigma_{e^{+} e^{-} \rightarrow \text { hadr }}(s) K(s) d s
$$

where the integral is carried out over the invariant mass squared $s$ of the hadronic system in the final state. The kernel $K(s)$ is a monotonous function behaving approximately like $1 / s$, while the annihilation cross section $\sigma_{e^{+} e^{-} \rightarrow h a d r}$ is being enhanced around the mass of the $\rho$ meson. The process $e^{+} e^{-} \rightarrow \pi^{+} \pi^{-}$below $1 \mathrm{GeV}$ contributes more than $60 \%$ to the total hadronic contribution [1]. Different measurements (KLOE [2] at the DAФNE accelerator, CMD-2 [3][4] at VEPP-2M) of the reaction $e^{+} e^{-} \rightarrow \pi^{+} \pi^{-}$for values of $\sqrt{s}$ around the $\rho$ meson peak have been performed and their results, together with the $\tau$ spectral function and $e^{+} e^{-}$data up to $3 \mathrm{GeV}$, have been used to produce a firm prediction [5] for the comparison with the direct measurement of the magnetic moment of the muon performed by E821 [6], at BNL. Comparing the theoretical values with the experimental results, one finds a deviation of $2.7 \sigma$ for the $e^{+} e^{-}$-based theoretical prediction, while the $\tau$-based values show only a $0.9 \sigma$ effect.

Different from other experiments, which measure the hadronic cross section in an energy scan, KLOE uses the the so-called radiative return method [7]. The centre-of-mass energy of the DA $\Phi N E$ collider is fixed at $\sqrt{s}=m_{\phi}=1020 \mathrm{MeV}$. The energy dependence of the cross section $e^{+} e^{-} \rightarrow$ hadrons can be obtained by using the radiative process $e^{+} e^{-} \rightarrow$ hadrons $+\gamma$, where the photon is radiated in the initial state, lowering in such a way the collision energy. The radiative cross section and the $\pi^{+} \pi^{-}$cross section are related through the radiator function:

$$
s_{\pi} \frac{d \sigma_{\pi^{+} \pi^{-} \gamma}}{d s_{\pi}}=\sigma_{\pi^{+} \pi^{-}}\left(s_{\pi}\right) \cdot H\left(s_{\pi}\right), s_{\pi}=M_{\pi \pi}^{2}
$$

\section{Small photon polar angles analysis}

KLOE has recently published an analysis which makes use of the radiative return method [2]. Events with two tracks from the interaction point, both having the polar angle between $50^{\circ}$ and $130^{\circ}$, are selected. No photons are detected but cuts on the missing momentum are applied, which correspond to polar photon angles $<15^{\circ}$ or $>165^{\circ}$. In this region (small polar photon angles) the Initial State Radiation (ISR in the following) process dominates over the Final State Radiation (FSR) process, where in the latter the photon is emitted from one of the two charged particles in the final state. Backgrounds from radiative bhabhas $e^{+} e^{-} \rightarrow e^{+} e^{-} \gamma$, muon pairs $e^{+} e^{-} \rightarrow \mu^{+} \mu^{-} \gamma$ and from the decay $\phi \rightarrow \pi^{+} \pi^{-} \pi^{0}$ is minimised for the given fiducial volume cuts. The rejection of the radiative bhabhas is performed by means of a likelihood method, based on the time of flight of the particles and on the energy released in the planes of the electromagnetic calorimeter. The other two background channels are rejected cutting on kinematical variables, built from the momenta of the pions. All the selection efficiencies are evaluated from data control samples, except the acceptance, which is estimated from MonteCarlo (PHOKHARA [8][9][10]). The same generator has been used to evaluate the radiator function $\mathrm{H}$, with an accuracy better than $1 \%$.

Once subtracted from the background and corrected for the efficiency, the spectrum has been normalised to the integrated luminosity of the sample (corresponding to $\sim 140 \mathrm{pb}^{-1}$ of 2001 data). 

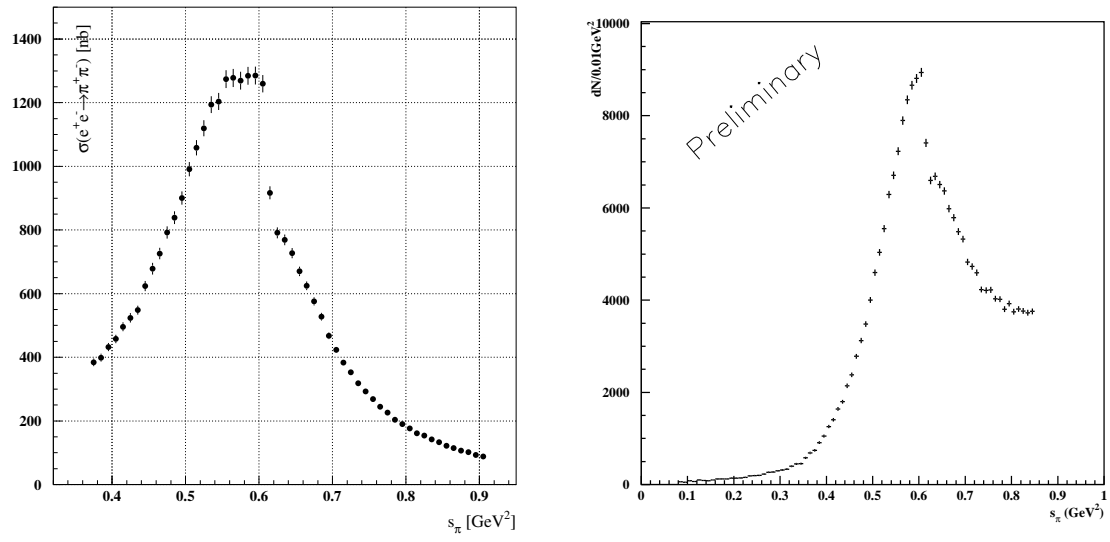

Figure 1: On the left: the cross section $\sigma\left(e^{+} e^{-} \rightarrow \pi^{+} \pi^{-}\right)$measured by KLOE using the radiative return method. The ISR photon has been emitted at small polar angles. On the right: $M_{\pi \pi}$ spectrum for the KLOE large photon angles analysis. The spectrum is not corrected for the acceptance.

Finally the spectrum has been divided by the radiator function $\mathrm{H}$, and corrected for FSR contribution [11][12] and the vacuum polarisation. The final result on $e^{+} e^{-} \rightarrow \pi^{+} \pi^{-}$cross section is shown in fig.1(left)

Integrating the cross section in the energy range $0.35<s_{\pi}<0.95 \mathrm{GeV}^{2}$, the following value (in $10^{-10}$ units) for the $\pi^{+} \pi^{-}$contribution to the muon anomaly $a_{\mu}^{\pi \pi}$ has been obtained:

$$
a_{\mu}^{\pi \pi}(0.35-0.95)=388.7 \pm 0.8_{\text {stat }} \pm 3.5_{\text {syst }} \pm 3.5_{t h} .
$$

\section{Large polar photon angles analysis}

A new and complementary analysis is now being performed at KLOE. Two tracks and at least one photon with the polar angle between $50^{\circ}$ and $130^{\circ}$ are required. This acceptance allows to explore the region at low $M_{\pi \pi}^{2}$ up to the threshold $4 m_{\pi}^{2}$, which is responsible for $\sim 20 \%$ of $a_{\mu}^{\text {hadr }}$ and which was kinematically suppressed in the small photon angle analysis. Moreover, it is required that the photon has an energy above $50 \mathrm{MeV}$, in order to exclude the region at high $M_{\pi \pi}^{2}$, where the background contribution from the decay $\phi \rightarrow f_{0}(980) \gamma \rightarrow \pi^{+} \pi^{-} \gamma$ is sizeable.

The main background contamination comes from the decay $\phi \rightarrow \pi^{+} \pi^{-} \pi^{0}$ : besides the requirements as in the previous work, two other cuts have been applied. A kinematic fit with the $\pi^{+} \pi^{-} \pi^{0}$ hypothesis (constraining the 4-momentum conservation and imposing $M_{\gamma \gamma}=m_{\pi^{0}}$ ) has been developed, excluding the events with low value of $\chi_{\pi \pi \pi}^{2}$. The $\pi^{+} \pi^{-} \pi^{0}$ events still left in the sample are finally rejected by a cut on the angle between the missing momentum and the tagged photon. A possible residual contamination from $\phi \rightarrow f_{0}(980) \gamma$ or $\phi \rightarrow f_{0}(600) \gamma$ has not been studied yet. The background subtracted $M_{\pi \pi}$ spectrum is shown in fig.1 (right).

The model dependence of FSR can be checked by observing the forward-backward asymmetry as a function $M_{\pi \pi}$ and comparing it with the MonteCarlo prediction within the model of scalar QED (point-like pions). The forward-backward asymmetry is defined as $A\left(M_{\pi \pi}\right)=\frac{N\left(\theta_{\pi^{+}}>90^{\circ}\right)-N\left(\theta_{\pi^{+}}<90^{\circ}\right)}{N\left(\theta_{\pi^{+}}>90^{\circ}\right)+N\left(\theta_{\pi^{+}}<90^{\circ}\right)}$ 


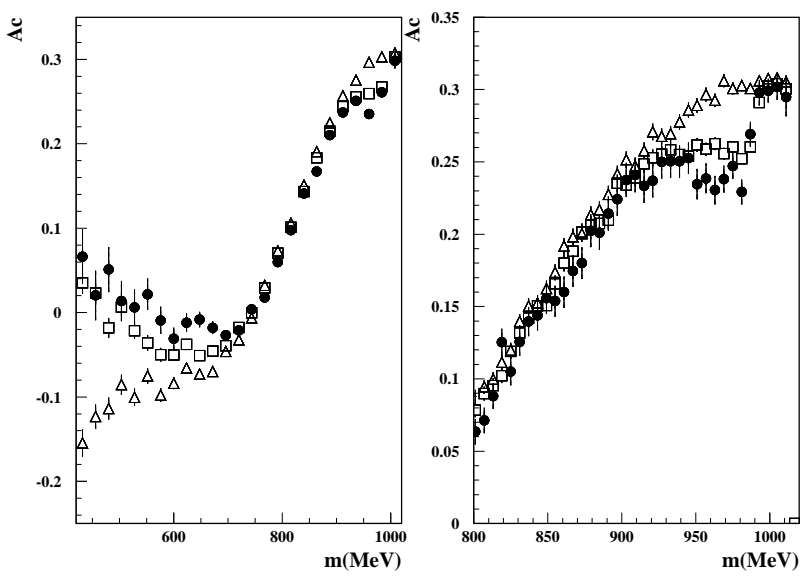

Figure 2: Forward-backward asymmetry. Full = data points, triangles = prediction ISR+FSR, squares = ISR+FSR+f0(980), this latter according to the kaon loop model. On the right, a zoom on the high $M_{\pi \pi}$ of the same plot.

and arises from the interference between ISR and FSR. It is very sensitive to the presence of scalar meson $f_{0}(980)$ [13], as can be seen in fig.2. Including the contribution of this particle (parametrised according the kaon loop model and using the final parameters as in [14]) we find a reasonable agreement between data and MonteCarlo.

\section{References}

[1] S.Eidelman, F.Jegerlenher, Z.Phys. C67 (1995) 585

[2] KLOE Collaboration, Phys.Lett B 606:12,24; 2005

[3] R. R. Akhmetshin et al., Phys. Lett. B 527 (2002) 161

[4] R. R. Akhmetshin et al., Phys. Lett. B 578 (2004) 285

[5] M. Davier, S. Eidelman, A. Höcker and Z. Zhang, Eur. Phys. J. C 31, 503 (2003)

[6] G. W. Bennett et al. Phys. Rev. Lett. 89 (2002) 101804 [Erratum-ibid. 89 (2002) 129903]

[7] S. Binner, J. H. Kühn and K. Melnikov, Phys. Lett. B 459, 279 (1999)

[8] G. Rodrigo, H. Czyż, J. H. Kühn and M. Szopa, Eur. Phys. J. C 24 (2002) 71

[9] J. H. Kühn and G. Rodrigo, Eur. Phys. J. C 25, 215 (2002)

[10] H. Czyż, A. Grzelińska, J. H. Kühn and G. Rodrigo, Eur. Phys. J. C 27, 563 (2003)

[11] J.S.Schwinger, Particles, Sources and Fields, Vol.3, Redwood City, USA Addison-Wesley (1989) 99

[12] A. Höfer, J. Gluza and F. Jegerlehner, Eur. Phys. J. C 24 (2002) 51

[13] H. Czyż, A. Grzelińska, J. H. Kühn, Phys.Lett. B 611 (2005) 116

[14] KLOE Collaboration; hep-ex/0511031 